Aplicación de luz UVC para esterilizar soluciones parenterales.

Artículo de Amasino, AJ.; Fernández Blanco, M.; Miranda, R.; Olivera, D.; Coll Cárdenas, F.

CIENCIA VETERINARIA, Vol. 20, № 2, julio-diciembre de 2018, ISSN 1515-1883 (impreso) E-ISSN 1853-8495 (en línea), pp. 13-26 DOI: http://dx.doi.org/10.19137/cienvet-201820201

\title{
Aplicación de luz UVC para esterilizar soluciones parenterales
}

\author{
Amasino, AJ. ${ }^{1}$; Fernández Blanco, M. ${ }^{1}$; Miranda, R. $^{2}$; Olivera, D. ${ }^{1,3}$; Coll Cárdenas, F. $^{1}$ \\ ${ }^{1}$ Cátedra de Biofísica, Facultad de Ciencias Veterinarias, Universidad Nacional de La Plata, \\ calle 60 y 118, s/n La Plata (1900). Pcia. de Bs. As. Argentina. \\ ${ }^{2}$ Hospital Escuela, Facultad de Ciencias Veterinarias, Universidad Nacional de La Plata. \\ ${ }^{3}$ Consejo Nacional de Investigaciones Científicas y Técnicas (CONICET). Argentina.
}

Dirección postal laboral: Calle 60 y 118 s/n, La Plata (1900). Buenos Aires.

Correo electrónico: aamasino@fcv.unlp.edu.ar

\section{RESUMEN}

Uno de los inconvenientes que suele presentarse al trabajar en un hospital veterinario es la necesidad de contar con un stock permanente de medicamentos y otros productos sanitarios, entre ellos, grandes volúmenes de soluciones parenterales estériles, las cuales son muy utilizadas en la clínica diaria. Los líquidos de reposición más frecuentemente empleados en medicina veterinaria son las soluciones de dextrosa al $5 \%$, las de cloruro de sodio al $0.9 \%$ y las de Ringer lactato, entre otras. Es fundamental que las soluciones a administrar se encuentren en condiciones de esterilidad. En ese sentido, la luz ultravioleta de onda corta (UVC), radiación no ionizante, es un poderoso agente bactericida que desinfecta sin alterar el color, sabor, olor o $\mathrm{pH}$ de la muestra. El objetivo de este trabajo fue estudiar la aplicación de radiación UVC para esterilizar soluciones parenterales con almacenamiento refrigerado.

Se trabajó con solución de dextrosa al 5 \% y solución fisiológica al $0.9 \%$ separadas en muestras control, sin tratar, y tratadas con luz UVC. La dosis de irradiación que recibieron las muestras fue de $3.4095 \mathrm{~J} /$ $\mathrm{cm}^{2}$. Posteriormente, fueron almacenadas en cámaras de refrigeración de temperatura controlada a $4^{\circ} \mathrm{C}$ durante 7 días. Se observó para el caso de las muestras irradiadas, que no se presentó desarrollo visible durante ese tiempo, conservando su esterilidad. Además, se determinó el factor de inactivación, F.I. \% al cabo de 7 días. En ambas soluciones estudiadas se obtuvieron entre un 99 a $100 \%$ de inactivación. 
Analizando los resultados obtenidos, el uso de la irradiación con luz UVC como medio de esterilización y el posterior mantenimiento de las soluciones a temperaturas de refrigeración, consideramos que constituye una interesante y nueva tecnología a implementar.

Palabras claves: Solución de dextrosa al 5 \%, solución fisiológica al $0.9 \%$, luz UVC, esterilización

\section{Application of UVC light to sterilize parenteral solutions}

\section{ABSTRACT}

One of the drawbacks that usually occurs when working in a veterinary hospital is the need to have a permanent stock of medicines and other health products, including large volumes of sterile parenteral solutions, which are widely used in the daily clinic. Replacement fluids most frequently used are the solutions of dextrose $5 \%$, sodium chloride $0.9 \%$ and Ringer lactate, among others. It is essential that the solutions to be administered are in sterile conditions. In that sense, short-wave ultraviolet light (UVC), non-ionizing radiation, is a powerful antibacterial agent that disinfects without altering the color, flavor, odor or $\mathrm{pH}$ of the sample.

The objective of this work was to study the application of UVC radiation to sterilize parenteral solutions with refrigerated storage. We worked with $5 \%$ dextrose solution and $0.9 \%$ physiological solution separated in control samples, untreated, and treated with UVC light. The irradiation dose received by the samples was $3.4095 \mathrm{~J} / \mathrm{cm} 2$. Subsequently, they were stored in temperature controlled refrigeration chambers at $4{ }^{\circ} \mathrm{C}$ for 7 days. It was observed for the case of the irradiated samples, that there was no visible development during that time, conserving its sterility. In addition, the inactivation factor, F.I. \%, was determined after 7 days. In both studied solutions , 99 to $100 \%$ inactivation was obtained. Analyzing the obtained results , the use of irradiation with UVC light as a means of sterilization and the subsequent maintenance of the solutions at refrigeration temperatures, we consider that it constitutes an interesting and new technology to be implemented.

Key words: $5 \%$ dextrose solution, $0.9 \%$ physiological solution, UVC light, sterilization

Fecha de recepción artículo original: 02-06-2018

Fecha de aceptación para su publicación: 13-11-2018 


\section{Introducción}

Uno de los inconvenientes que suele presentarse al trabajar en un hospital veterinario es la necesidad de contar con un stock permanente de medicamentos y otros productos sanitarios, entre ellos, grandes volúmenes de soluciones parenterales estériles, las cuales son muy utilizadas en la clínica diaria. Diversas situaciones hacen que en más de alguna ocasión este suministro se vea interrumpido, lo cual repercute negativamente tanto en los propósitos de la terapéutica clínica, como en el interés de los propietarios. ${ }^{(1)}$

El agua y los electrolitos del organismo se encuentran distribuidos en distintos compartimentos en constante equilibrio. El mayor volumen forma parte del líquido intracelular (LIC), constituyendo el $60 \%$ del agua corporal total (ACT), mientras que el volumen del líquido extracelular (LEC) es del $40 \%$. De ellos, el $32 \%$ constituye el volumen del líquido intersticial y sólo el $8 \%$ representa el volumen sanguíneo (volemia), estimándose el volumen plasmático alrededor de 35-40 ml $/ \mathrm{kg}^{(2)}$. El Na+ es el catión más importante dentro del contenido del LEC ya que se encarga del desarrollo de la presión osmótica del plasma y de mantener el volumen de este líquido. Alteraciones en este catión conllevan a manifestaciones clínicas como: edema cerebral, deshidratación, ceguera, ataxia. La hiponatremia es el resultado de una pérdida importante del ión $\mathrm{Na}^{+}$, que ocurre en enfermedades como obstrucciones intestinales, enterocolitis, enfermedad renal. ${ }^{(3)} \mathrm{El}$ ion $\mathrm{Cl}^{-}$también está presente en altas concentraciones en el LEC, por lo que sus variaciones resultan en cambios grandes a nivel corporal. En la mayoría de los sitios del cuerpo, el $\mathrm{Cl}^{-}$tiende a seguir pasivamente al $\mathrm{Na}^{+}$por difusión a través de la membrana celular, por lo que dentro del LEC las relaciones de estos dos iones son directas $\left({ }^{4 ; 3)}\right.$. La hipocloremia ocurre como resultado de la gran pérdida de este anión, en situaciones clínicas como enfermedades gastrointestinales, obstrucciones, íleo paralítico, enteritis proximal, estando acompañadas, en la mayoría de los casos de alcalosis metabolic ${ }^{(4)}$.

Por otro lado, la glucosa es el monosacárido más importante para todos los organismos vivos, constituyendo su principal fuente de energía. Forma parte de un 0.08-0.1 \% del contenido sanguíneo de todos los mamíferos normales. Es la precursora de la síntesis de todos los demás carbohidratos en el cuerpo, incluidos el glucógeno para almacenamiento, la ribosa y desoxirribosa en los ácidos nucleicos, la galactosa, los glucolípidos y, en combinación con las proteínas, las glucoproteínas y los proteoglucanos. ${ }^{(5,6)}$ En los animales, la regulación de la concentración del azúcar en sangre es esencial para el organismo, 
ya que estados de hiperglucemia o hipoglucemia extremos pueden ser críticos para la supervivencia del animal.

En la mayoría de estas situaciones clínicas, en las cuales se ve alterado el equilibrio hidroelectrolítico en el organismo animal, la fluidoterapia tiene como objetivos: a) reemplazar la deficiencia de fluidos y/o b) suplir el requerimiento del mantenimiento de los mismos ${ }^{(7)}$ Las soluciones parenterales constituyen así, uno de los pilares terapéuticos más utilizados en la práctica diaria de la medicina veterinaria. El tipo de solución a emplear dependerá del compartimento que se desee reponer, por ejemplo, la hidratación y reposición de electrolitos del líquido intersticial se debe realizar con cristaloides isotónicos; para ello se deben valorar las pérdidas insensibles debidas a la exposición quirúrgica (0.5-1 ml $/ \mathrm{kg} / \mathrm{h}$ ) y el volumen de dieresis ${ }^{(2)}$.

Los líquidos de reposición más frecuentemente empleados en medicina veterinaria son las soluciones de dextrosa al $5 \%$, las de cloruro de sodio al $0.9 \%$ y las de Ringer lactato, entre otras. Particularmente, la solución de dextrosa al $5 \%$ (252 mOsm) contribuye a la restauración de los niveles sanguíneos de glucosa, minimiza el gasto de glucógeno hepático y disminuye la degradación de proteínas como fuente de energía, por lo cual la misma es empleada no sólo en casos de deshidratación sino también en casos de hipoglucemia e hipovolemia. Puede administrarse por vía parenteral, principalmente intraveno$\mathrm{sa}^{(8)}$, siendo utilizada también como diluyente medicamentoso.

La solución fisiológica de cloruro de sodio al $0.9 \%$ es una de las principales soluciones parenterales, ya que es isotónica con respecto al plasma sanguíneo, es el cristaloide más simple y está equilibrado en cargas positivas y negativas ${ }^{(9)}$, siendo por tanto, uno de los más utilizados para la restitución de fluidos por vía parenteral tanto en pequeños como en grandes animales. Además de su aplicación para la restitución de fluidos, la solución fisiológica es de utilidad en la realización de lavajes broncoalveolares en equinos, por ejemplo, o para la toma de muestras en estudios citológicos. En este último caso, cuando la efusión abdominal es muy escasa o no se puede obtener, o bien se sospecha de neoplasia abdominal, se puede hacer el lavado peritoneal ${ }^{(10)}$.

Es fundamental que las soluciones a administrar se encuentren en condiciones de esterilidad ya que de lo contrario, según la vía de administración, podría desarrollarse una infección subcutánea, una peritonitis (en el caso del lavado peritoneal), y, como complicación más grave, una septicemia fulminante.

Para esterilizar estas soluciones generalmente se utiliza calor húmedo o filtración, pero ambos procesos resultan caros y dificultosos a la hora de requerir grandes volúmenes, como es el caso de la medicina 
veterinaria de equinos. ${ }^{(11)}$ Además, Montes González et al. ${ }^{(12)}$, mencionan que el proceso de esterilización en una autoclave puede resultar problemático en algunas ocasiones, ya que puede requerir mucho tiempo, parte del líquido puede llegar a ebullición y evaporarse e incluso no siempre se logra saber si el contenido del interior de los frascos ha alcanzado la temperatura requerida de esterilización (por ejemplo, $121^{\circ} \mathrm{C}$ ), considerando también que se deben manejar bajos volúmenes de solución.

Teniendo en cuenta dichas limitaciones, resulta interesante indagar acerca de la aplicación de tecnologías alternativas que permitan obtener un alto poder de descontaminación y un menor costo que la esterilización convencional aplicada en la actualidad.(13)

En ese sentido, la luz ultravioleta de onda corta (UVC), radiación no ionizante, es un poderoso agente bactericida ya que al ser absorbida por las proteínas y ácidos nucleicos, afecta al material genético de los microorganismos, induciendo cambios en la multiplicación y viabilidad celular. ${ }^{(14)}$ Esta tecnología se ha aplicado exitosamente en la inactivación de microorganismos que contaminan el agua y superficies de diversos materiales. ${ }^{(15)}$ Se usa para la desinfección de grandes cantidades de microorganismos en aguas potables y residuales ${ }^{(16)}$ y además en la industria alimenticia, farmacéutica y en hospitales se utiliza para esterilización de superficies, utensilios, aire y también material de empaque. ${ }^{(17)}$ Debido a que la radiación UVC desinfecta sin alterar el color, sabor, olor o $\mathrm{pH}$ de la muestra tratada, es un medio eficaz para garantizar que el agua sea microbiológicamente segura; el criterio de funcionamiento normal se basa en una reducción del $99.999 \%$ de los microorganismos. Además, tiene como ventajas que no deja residuos y tampoco altera su composición o propiedades. Como desventajas podemos mencionar su bajo poder de penetración y efectividad en el caso de presencia de contaminantes o turbidez en el agua ${ }^{(16)}$.

Por otro lado, es conocido que la temperatura de refrigeración modifica el desarrollo bacteriano ya que a medida que desciende, el crecimiento se hace más lento hasta que finalmente se detiene.

Por lo expuesto, el objetivo de este trabajo fue estudiar la aplicación de radiación UVC para esterilizar soluciones parenterales. Se planteó además, mantener dichas soluciones en almacenamiento refrigerado y estudiar dicho efecto.

\section{Materiales y métodos}

Se trabajó con dos soluciones diferentes: solución de dextrosa al 5 $\%$ (experiencia I) y solución fisiológica al 0.9 \% (experiencia II). 
Las mismas se prepararon asépticamente a partir de agua destilada, obtenida por ósmosis inversa mediante el destilador S-5/240BTD (Silicon Química SRL) y dextrosa anhidra (Biopack) (experiencia I) y cloruro de sodio (Anedra, Research AG SA) (experiencia II).

Experiencia I: la solución glucosada se preparó a partir de 25 gramos de dextrosa a la cual se le agregó agua destilada hasta completar un volumen de $500 \mathrm{ml}$, en bolsas transparentes estériles, de 51 micrones de espesor, las cuales fueron posteriormente termoselladas en el extremo abierto y se homogenizó dicha solución por agitación. Se prepararon un total de 12 bolsas las que fueron separadas en dos lotes; unas consideradas como control, sin tratar, y otras tratadas con luz UVC.

Experiencia II: este caso correspondió a la solución fisiológica al $0.9 \%$, la cual se obtuvo a partir de 4.5 gramos de cloruro de sodio al que se le agregó agua destilada hasta completar un volumen de 500 $\mathrm{ml}$, siguiendo posteriormente el mismo procedimiento que para la experiencia I.

Para el tratamiento de esterilización de las muestras, experiencia I y II, se procedió a irradiar ambas caras de las bolsas con luz UVC durante un tiempo total de 30 minutos (15 minutos de cada lado), utilizando un equipo diseñado para tal fin, que consta de dos tubos de luz UVC (de 15 watts c/u y de $45 \mathrm{~cm}$ de longitud, marca Philips) y estantes ubicados a una distancia de $15 \mathrm{~cm}$ de altura. La dosis de irradiación total que recibieron las muestras fue de $3.4095 \mathrm{~J} / \mathrm{cm}^{2}$ determinada mediante el empleo de un radiómetro digital (marca Cole Parmer) Figura 1.
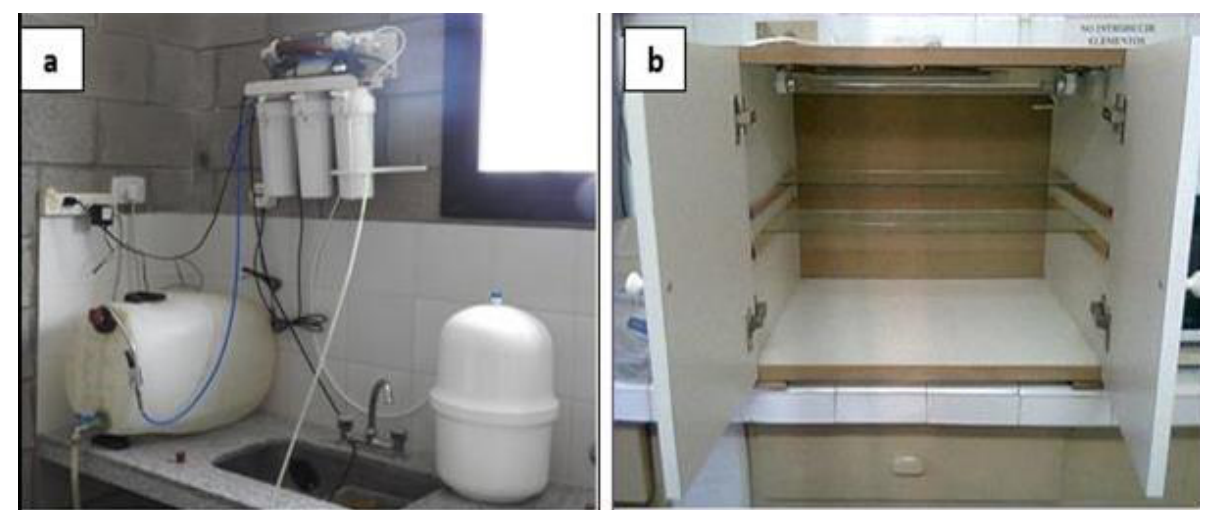

Figura 1. Equipos utilizados para la preparación de las muestras. a) Destilador de agua de ósmosis inversa; b) Equipo de irradiación con luz UVC. (Fuente propia) 
Posteriormente, las bolsas fueron almacenadas en cámaras de refrigeración de temperatura controlada a $4^{\circ} \mathrm{C}$ durante 7 días.

A diferentes tiempos de almacenamiento, se realizó la siembra en profundidad de $1 \mathrm{ml}$ de las muestras tratadas y control, por separado, utilizando como medio de cultivo Agar Plate Count. Las placas fueron incubadas en estufa de cultivo a $37^{\circ} \mathrm{C}$, por 24 horas, tiempo al cabo del cual se realizó el recuento correspondiente para determinar Microorganismos Aerobios Mesófilos Totales.

Las determinaciones se realizaron por duplicado y los resultados fueron expresados como $\log \mathrm{N}$ (N: Unidades Formadoras de Colonias $\left.\mathrm{ml}^{-1}\right)$.

\section{Análisis estadístico}

La cinética del crecimiento se modeló empleando modelos matemáticos mediante el software estadístico Sigma Plot 11.0 (Systat Software Inc.). Se ajustaron los datos obtenidos a partir del modelo de Gompertz, calculando el conjunto de parámetros con la menor suma residual de cuadrados y un intervalo de confianza del $95 \%$. Además, el software ofrece para cada ajuste de los datos, la suma de cuadrados, el grado de libertad (DF) y el cuadrado medio debido a la regresión y la variación residual. En otros casos también fue posible utilizar el modelo de regresión lineal, particularmente cuando el número de microorganismos en las soluciones permaneció constante o disminuyó durante el almacenamiento. ${ }^{(18)}$

Un diseño factorial fue utilizado para analizar el efecto de la luz UVC aplicada como agente físico esterilizante de las soluciones parenterales de estudio, mantenidas a temperatura de refrigeración. Las experiencias para ambas soluciones se realizaron por duplicado utilizando simultáneamente muestras tratadas y sin tratar, control $(2 \times 2 \times 2)$.

\section{Resultados y discusión}

En primer lugar, para la solución de la experiencia I, se observó que la muestra sin irradiar, control, presentó un recuento microbiano inicial de $2.49 \log$ UFC ml-1 , aumentando hasta 1.65 veces su valor al cabo de los 7 días que duró la experiencia. En tanto para el caso de las muestras irradiadas, no se presentó desarrollo visible durante ese tiempo, conservando su esterilidad. Dichos resultados se presentan en la Figura 2. 


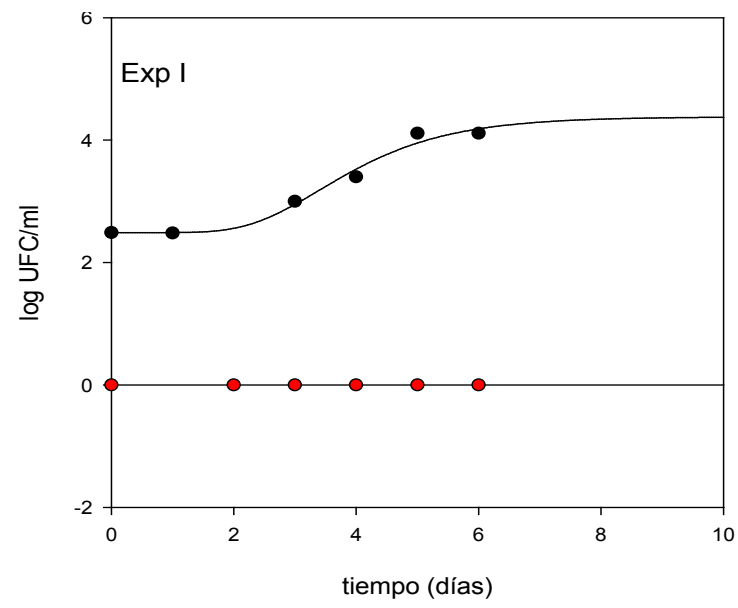

Figura 2. Recuento de Microorganismos Aerobios Mesófilos Totales en muestras de solución glucosada (experiencia I). (•) Muestras control; (•) Muestras tratadas. Las líneas curvas muestran la aplicación del Modelo de Gompertz; las líneas rectas, el modelo de regresión lineal.

La cinética del crecimiento microbiano de las muestras control pudo ser analizada aplicando el modelo matemático de Gompertz, en tanto en las tratadas, al no observarse desarrollo durante el tiempo de estudio se debió utilizar el modelo de regresión lineal (Figura 2). Los parámetros derivados de ambos modelos se presentan en la Tabla 1.

Tabla 1. Parámetros de modelos matemáticos que predicen el desarrollo de Microorganismos Aerobios Mesófilos Totales en muestras de solución glucosada, experiencia I.

\begin{tabular}{|c|c|c|c|c|c|c|}
\hline \multicolumn{4}{|c|}{ Parámetros de Gompertz } & \multicolumn{3}{|c|}{ Parámetros derivados } \\
\hline a & c & $\mathrm{b}$ & $\mathrm{m}$ & $\mu$ & LPD & MPD \\
\hline \multicolumn{7}{|c|}{ Muestras Control } \\
\hline $2.48 \pm 0.11$ & $1.89 \pm 0.43$ & $0.85 \pm 0.46$ & $3.40 \pm 0.36$ & 0.59 & 2.22 & 4.37 \\
\hline \multicolumn{7}{|c|}{ Muestras Tratadas } \\
\hline ------------- & ------------ & ------------ & ------------ & 0.00 & 25 & R2: 0.80 \\
\hline
\end{tabular}

a, (log UFC ml-1); c, (log UFC $\left.\mathrm{ml}^{-1}\right)$; b, (días) ${ }^{-1} ; \mathrm{m}$, (días); velocidad específica de crecimiento $(\mu)$, (log UFC $\mathrm{ml}^{-1}$ día $\left.^{-1}\right)$; máxima densidad de población (MPD), (log UFC $\mathrm{ml}^{-1}$ ); duración de fase de latencia (LPD), (días); ----, indica la aplicación del modelo de regresión lineal. 
Analizando los parámetros presentados en la Tabla 1 puede observarse que en las muestras consideradas control, los microorganismos tardaron aproximadamente dos días en desarrollar, al presentar una fase de latencia (LPD) de 2.22 días, en tanto en el caso de las muestras irradiadas, este valor aumentó 11.26 veces (25 días). En relación al parámetro MPD (máxima densidad poblacional), en el primero de los casos alcanzó un valor final de $4.37 \log \mathrm{UFC} \mathrm{m}{ }^{-1}$ al cabo de los 7 días que duró la experiencia, en tanto en las muestras irradiadas este valor no pudo ser calculado debido a no evidenciarse desarrollo bacteriano, teniendo el tratamiento acción germicida. En este caso, se presentó un muy buen coeficiente de determinación $\left(\mathrm{R}^{2}\right)$ confirmando el orden de la reacción de inactivación de las bacterias ${ }^{(19)}$ Figura 3.

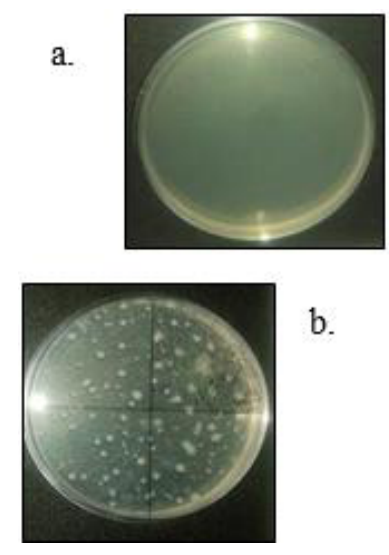

Figura 3. Desarrollo en Agar Plate Count. a. Recuento de Microorganismos Aerobios Mesófilos Totales en muestras tratadas con luz UVC; b. Recuento de Microorganismos Aerobios Mesófilos Totales en muestras control (sin tratar).

La Figura 4 muestra los resultados obtenidos en la experiencia II. Puede observarse que las muestras control alcanzaron un valor final de $4.86 \log \mathrm{UFC} \mathrm{ml}^{-1}$, en tanto en las muestras tratadas no se presentó desarrollo durante todo el período de tiempo que duró la experiencia, al igual que en la solución correspondiente a la experiencia I. Asimismo, en la Tabla 2 se presentan los parámetros obtenidos a partir de la aplicación de los modelos matemáticos. En concordancia con la experiencia I, las muestras control fueron modeladas a partir de la ecuación de Gompertz, en tanto las muestras tratadas debieron ser modeladas a partir de una regresión lineal al no presentar desarrollo durante el tiempo de estudio (7 días).

En ambos casos se observó muy buena correlación entre los resultados predichos por el modelo y los datos experimentales. 


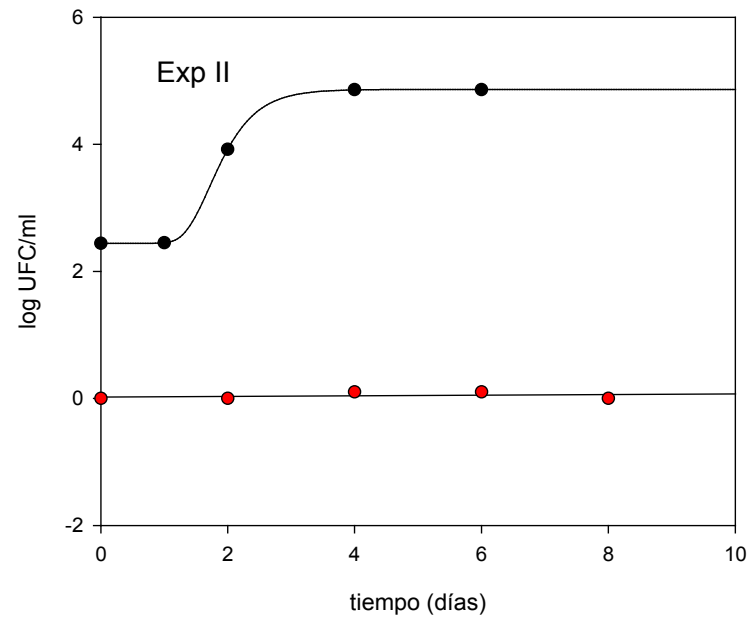

Figura 4. Recuento de Microorganismos Aerobios Mesófilos Totales en muestras de solución fisiológica (experiencia II). (•) Muestras control; (•) Muestras tratadas. Las líneas curvas muestran la aplicación del Modelo de Gompertz; las líneas rectas, el modelo de regresión lineal.

Tabla 2. Parámetros de los modelos matemáticos que predicen el desarrollo de Microorganismos Aerobios Mesófilos Totales en muestras de solución fisiológica, Experiencia II.

\begin{tabular}{|c|c|c|c|c|c|c|}
\hline \multicolumn{4}{|c|}{ Parámetros de Gompertz } & \multicolumn{3}{|c|}{ Parámetros derivados } \\
\hline $\mathbf{a}$ & c & b & $\mathbf{m}$ & $\mu$ & LPD & MPD \\
\hline \multicolumn{7}{|c|}{ Muestras Control } \\
\hline $2.44 \pm 0.01$ & $2.42 \pm 0.01$ & $2.48 \pm 0.21$ & $1.87 \pm 0.02$ & 2.20 & 1.46 & 4.86 \\
\hline \multicolumn{7}{|c|}{ Muestras Tratadas } \\
\hline ------------- & ----------- & ----------- & ----------- & 0.02 & 25 & $\mathrm{R}^{2}: 1$ \\
\hline
\end{tabular}

a, $\left(\log \mathrm{UFC} \mathrm{ml}^{-1}\right) ; \mathrm{c},\left(\log \mathrm{UFC} \mathrm{ml}^{-1}\right) ; \mathrm{b}$, (días$^{-1} ; \mathrm{m}$, (días); velocidad específica de crecimiento $(\mu),\left(\log \mathrm{UFC} \mathrm{ml}^{-1}\right.$ día $\left.^{-1}\right)$; máxima densidad de población (MPD), (log UFC $\mathrm{ml}^{-1}$ ); duración de fase de latencia (LPD), (días); ----, indica la aplicación del modelo de regresión lineal.

Analizando los parámetros derivados de la aplicación de los modelos se puede inferir que la velocidad específica de crecimiento $(\mu)$ fue 110 veces superior en el caso de las muestras control con respecto a las tratadas, las cuales presentaron una fase de latencia (LPD) de 25 días (resultado más de 17 veces mayor que para las muestras sin tratar). Al igual que en la experiencia anterior, en dichas muestras, el coeficiente de determinación $\mathrm{R}^{2}$ fue óptimo. 
El tiempo de inactivación conseguido en el presente estudio resultó mayor al informado en un trabajo previo ${ }^{(20)}$ en el cual muestras de solución fisiológica irradiadas con luz UVC y almacenadas a temperatura ambiente, no presentaron desarrollo microbiano durante los primeros dos días de la experiencia, remarcando de esta manera la importancia del almacenamiento refrigerado de las muestras para asegurar su esterilidad por un periodo más prolongado de tiempo.

Por último, se determinó el factor de inactivación, F.I. \%, a partir de los promedios de los recuentos finales alcanzados en cada experiencia al cabo de 7 días, tanto para las muestras control como para las tratadas utilizando la siguiente formula ${ }^{(21)}$ :

$$
\text { F. I. } \%=\frac{U c-U n p}{U c} \times 100
$$

Donde Uc es el promedio de los recuentos finales de las muestras control y Unp el promedio de los recuentos finales para las muestras tratadas.

Estos valores mostraron que para ambas soluciones estudiadas se obtuvieron entre un 99 a 100\% de inactivación, resultando promisorios y siendo consistentes con los presentados por Pantoja-Espinoza et al. ${ }^{(19)}$, quienes trabajando con efluentes en una planta depuradora de agua obtuvieron igual índice de inactivación bacteriana por fotolisis UV, coincidiendo también, con la reducción microbiana observada por Bintsis et al. ${ }^{(16)}$.

\section{Conclusiones}

Analizando los resultados obtenidos y teniendo en cuenta la frecuente utilización de grandes volúmenes de soluciones parenterales como dextrosa al $5 \%$ y cloruro de sodio al $0.9 \%$, con diversos fines terapéuticos en la clínica médica veterinaria, es necesario contar con un sistema de esterilización que aplicado a las mismas resulte rápido, eficaz, económico y pueda ser empleado en grandes cantidades. Con tal fin, el uso de la irradiación con luz UVC como medio de esterilización y el posterior mantenimiento de las soluciones a temperaturas de refrigeración, consideramos que constituye una interesante y nueva tecnología a implementar. 


\section{Bibliografía}

1. Delgado Quintanilla, P. Elaboración de un manual de métodos alternos para la preparación de soluciones parenterales de gran volumen de uso hospitalario. Tesis de grado, Universidad de El Salvador, Facultad de Química y Farmacia. 2002.

2. -Uña Orejón, R.; Gisbert de la Cuadra, L.; Garríguez Pérez, D.; Díez Sebastián, J.; Ureta Tolsada, MP. Fluidoterapia de mantenimiento administrada en un hospital terciario: estudio de prevalencia. Revista Española de Anestesiología y Reanimación. 2017. 64(6):306-312.

3. Reed, S.; Warwick, B.; Sellon, D. Equine internal medicine, 2nd ed., Saunders, Philadelphia.2004; 1566 p.

4. Coumbe, K. Equine Veterinary Nursing, 2nd ed., Wiley-Blackwell, London, 2001; 496 p.

5. Cazco Pérez, D. Utilidad del péptido C y la hemoglobina glicosilada en el diagnóstico y control de terapia de pacientes diabéticos tipo 2 del Hospital Provincial General Docente Riobamba. Tesis de grado. Escuela Superior Politécnica de Chimborazo. Facultad de Ciencias, Escuela de Bioquímica y Farmacia. 2012.

6. Guyton, A.; Hall, J. Tratado de fisiología Médica, 13ra ed., Elsevier, Madrid; 2016. 1168 p.

7. Robinson, E.Current Therapy in equine medicine, Saunders, Philadelphia; 2003. 960 p.

8. Dvorkin, M.; Cardinali, D.; Ierrmoli, R. Best \&Taylor. Bases Fisiológicas de la Práctica Médica, 14ta ed., , Médica Panamericana, Buenos Aires; 2014. 1164 p.

9. Moral, V.; Colilles, C.; Camps, A.; Basora, M.; Galán, J. Debates actuales en fluidoterapia. Info colloids extra. Barcelona. Fresenius Kabi España, S.A.U.; 2008. 24 p.

10. del Amo, AN.; Diessler, M. Estudio citológico de los fluidos torácico y abdominal. En: Décimo tercer curso sobre citología diagnóstica en pequeños animales; La Plata. Facultad de Cs Veterinarias, UNLP; 2015.p. 37-40.

11. Miranda, R.; Amasino, A.; Olivera, D.; Coll Cárdenas, F. Soluciones Fluidoterápicas: Esterilización Mediante Nuevas Tecnologías. En: X Jornadas Internacionales de Veterinaria Práctica; Mar del Plata, 2017 (Consultado 11/10/2018). Disponible en: http:// cvpba.org/wp-content/uploads/2017/09/3.CB .POSTER2017.pdf.

12. Montes-González, Y.; Hidalgo-Guerrero, L.; Mayo-Abad, O. Esterilización de soluciones para productos parenterales. Análisis de la problemática. Revista Tecnología Química. 2017. 37 (3): 367-379.

13. Prietobon Tarrán, E; Desinfección por luzultravioleta. 2002 (Consultado 10/10/2018). Disponible en: http://www.calidadambiental14000.com/cursos/uv.pdf.

14. Haughton, P.; Lyng, J.; Cronin, D.; Morgan, D.; Fanning, S.; Whyte, P. Efficacy of UV light treatment for the microbiological decontamination of chicken, associated packaging, and contact surfaces. Journal of Food Protection. 2011. 74 (4): 565-572. 
15. López Díaz, AS.; Palou, E.; López Malo, A. Radiación ultravioleta en jugos de frutas: fundamentos y aplicaciones. Temas selectos de Ingeniería de Alimentos. 2012. 6 (2):79-93.

16. Bintsis, T.; Litopoulou-Tzanetaki, E.; Robinson, R. Existing and potential applications of ultraviolet light in the food industry - a critical review. Journal of the Science of Food and Agriculture. 2000. 80: 637-645.

17. Schenk, M. 2010. Preservación de productos frutales mínimamente procesados mediante la aplicación de luz UV y su combinación con otras tecnologías emergentes. Tesis Doctoral, FCEN, UBA (Consultado 10/10/2018). Disponible en: http://digital. bl.fcen.uba.ar/download/tesis/tesis n4717_Schenk.pdf.

18. Coll Cárdenas, F.; Giannuzzi, L.; Zaritzky, N. Modelling microbial growth in meat broth with added lactic acid under refrigeration storage. International Journal of Food Science and Technology. 2007. 42:175-184.

19. Pantoja-Espinoza, JC.; Proal-Nájera, JB.; García-Roig, M.; Cháirez-Hernández, I.; Osorio-Revilla, GI. Eficiencias Comparativas de Inactivación de Bacterias Coliformes en efluentes municipales por fotolisis (UV) y por fotocatálisis $\left(\mathrm{UV} / \mathrm{Tio}_{2} / \mathrm{Sio}_{2}\right)$. Caso: Depuradora de aguas de Salamanca, España. Revista Mexicana de Ingeniería Química. 2015. 14 (1):119-135.

20. Fernández Blanco, M.; Miranda, R.; Olivera, D.; Coll Cárdenas, F. Uso de nuevas alternativas para la esterilización de soluciones fluidoterápicas. En: 9nas Jornadas Internacionales de Veterinaria Práctica; Mar del Plata, 2015 (Consultado 11/10/2018). Disponible en: http://cvpba.org/wp-content/uploads/2015/09/1.ciencias basicas. pdf

21. Prieto Serratos, EA.; Gutiérrez Landa, R.; Rivera, AL.; Arredondo de la Rosa, MC.; Fernández Escobar, F. Efectos bactericidas de pulsos concentrados de radiación electromagnética. En: CIINDET 2010, VIII Congreso Internacional sobre Innovación y Desarrollo Tecnológico; Cuernavaca, México 2010 (Consultado 10/10/2018). Disponible en: https://www.researchgate.net/publication/267639990. 
